\title{
Rheophysical study of dispersed alumina suspensions
}

\author{
Florian Boutenel $^{\mathrm{a}, *}$, Gilles Dusserre $^{\mathrm{a}}$, Anne Aimable ${ }^{\mathrm{b}}$, Thierry Chartier ${ }^{\mathrm{b}}$, Thierry Cutard ${ }^{\mathrm{a}}$ \\ a ICA (Institut Clément Ader), Université de Toulouse, CNRS, IMT Mines Albi, INSA, UPS, ISAE-SUPAERO, Campus Jarlard, 81013 Albi, CT Cedex 09, France \\ ${ }^{\mathrm{b}}$ IRCER - Université de Limoges, CNRS, UMR 7315, Centre Européen de la Céramique, 12 Rue Atlantis, 87068 Limoges, France
}

Keywords:

Alumina suspensions

Dispersion

Rheology

Maximum packing fraction

Rheophysical classification

\section{A B S T R A C T}

This paper studies the rheological behavior of aqueous suspensions of fine grained $\left(\mathrm{d}_{50}=200 \mathrm{~nm}\right)$ alumina. Rheological measurements were performed on suspensions containing various amounts of solid and $0.26 \mathrm{wt} \%$ of ammonium polymethacrylate in order to ensure a good state of dispersion. Brownian motions, colloidal interactions and hydrodynamic interactions dictate the behavior. The viscosity is mainly influenced by the shear rate and by the solid volume fraction. A Maron and Pierce model was used to describe this last effect in the hydrodynamic regime. The maximum packing fraction was found to be $40 \mathrm{vol} \%$. This value is related to the colloidal stability as described by the DLVO theory. In fact, the maximum packing fraction leads to a surface-to-surface separation distance of about $46 \mathrm{~nm}$ because of the repulsive potential. Finally, a dimensionless approach was achieved to quantitatively identify, on the rheogram, the different flow regimes associated to each dominant interaction

type.

\section{Introduction}

Colloidal processing represents a preferred way to produce high performance ceramic materials [1-3]. All methods related to this concept, such as slip casting, pressure casting, tape casting, gelcasting or suspensions for spray-drying granules for die-pressing, start with a suspension in which ceramic particles are dispersed in a liquid, generally in aqueous medium. Furthermore, colloidal processing involves the control of the interparticle forces in order to limit heterogeneities and to optimize suspension properties. Indeed, a good state of dispersion of particles in a concentrated suspension is often a prerequisite to obtain high green density and a homogeneous particle arrangement, which in turn influences the sinterability and the properties of the final product $[4,5]$. In particular, alumina particles are frequently prepared as aqueous suspensions for colloidal processing [6].

Interparticle interactions can be attractive or repulsive. The Derjaguin - Landau - Verwey - Overbeek (DLVO) theory [7,8] establishes the total potential energy between a pair of charged particles. Two major interactions are considered. On the one hand, the attractive Van der Waals forces are mainly due to the interaction of instantaneous dipoles generated within the atoms comprising each particle. On the

\footnotetext{
* Corresponding author at: ICA (Institut Clément Ader), Université de Toulouse, CNRS, IMT Mines Albi, INSA, UPS, ISAE-SUPAERO, Campus Jarlard, 81013 Albi, CT Cedex 09, France.

E-mail addresses: florian.boutenel@mines-albi.fr (F. Boutenel), gilles.dusserre@mines-albi.fr (G. Dusserre), anne.aimable@unilim.fr (A. Aimable), thierry.chartier@unilim.fr (T. Chartier), thierry.cutard@mines-albi.fr (T. Cutard).
}

other hand, the repulsive electrical double layer forces result from the presence of surface charges on the particles. In a dispersed suspension, the total potential should remain repulsive in order to prevent aggregation. Experimentally, the zeta potential $\zeta$, which is the electrical potential in the interfacial double layer at the location of the slipping plane, is measured. An absolute value greater than $30 \mathrm{mV}$ commonly defines a stable suspension [9]. The electrical double layer forces can also be easily manipulated in order to provide repulsion between particles and overcome Van der Waals forces. Because the metal oxide surface is amphoteric, the surface charges can be modified by adjusting the suspension $\mathrm{pH}$. However, the addition of a dispersant is particularly effective in an aqueous medium. A wide variety of dispersant is commercially available. Furthermore, polyelectrolytes and polymers are largely used as electrosteric stabilizers in the literature. Due to its adsorption onto the surface of particles, the dispersant can modify the surface charges. Moreover, the involved steric effect can also help to promote the repulsion between particles.

The rheological behavior of suspensions determines the processability of these materials. However, it depends on the chemical and physical properties of the suspensions [10-13]. Thus, those properties should be optimized in order to obtain the desired flow during the process [14-16]. In consequence, understanding the rheological behavior of suspensions is essential.

Coussot and Ancey [17] have proposed a rheophysical classification of suspensions. Different flow regimes can be distinguished as a function of shear rate and solid volume fraction and each of them is associated to a dominant interaction type: Brownian motions, colloidal interactions, hydrodynamic interactions, friction, lubrication, collisions and turbulence. 
Furthermore, the viscosity $\eta$ of a suspension is strongly dependent on its solid volume fraction $\varphi$. In order to describe this effect for spherical particles, simple relations such as the one derived by Einstein $[18,19]$ can be applied:

$\eta=\eta_{0} \cdot(1+2.5 \cdot \varphi)$

where $\eta_{0}$ is the viscosity of the interstitial fluid. However, for solid volume fraction higher than $5 \mathrm{vol} \%$, the viscosity deviates significantly from this equation. Thus, physicist have proposed various empirical expressions like Maron and Pierce [20]:

$\eta=\eta_{0} \cdot\left(1-\frac{\varphi}{\varphi_{\max }}\right)^{-2}$

The maximum packing fraction $\varphi_{\max }$ is defined as the solid volume fraction for which the viscosity mathematically diverges. It depends on the arrangement taken by the particles. Thus, $\varphi_{\max }$ is affected by the particle size distribution and by the particle shape and roughness. In addition, this single parameter expresses the influence of various effects such as the dependence on the applied shear rate [21]. For monosized spherical particles, $\varphi_{\max }$ is approximately equal to 0.64 (random packing) at low shear rates whereas it is equal to 0.74 (facecentered cubic) at high shear rates. Many authors have proposed alternative expressions. For instance, Krieger-Dougherty [22] used a similar form where the exponent is equal to $-[\eta] \varphi_{\max }$, with $[\eta]$ the intrinsic viscosity depending on the particle shape. However, Barnes et al. [23] showed that this product is centered around -2 for many diverse situations.

The aim of the present work is to study the rheological behavior of dispersed alumina suspensions. After determining the optimum concentration of dispersant agent that ensures a good state of dispersion, rheological measurements are performed on alumina suspensions with various solid volume fractions. The rheological behavior is then described. This paper offers two significant new physicochemical understandings of alumina suspensions. On the one hand, a link between the rheology and the interparticle interactions is established. Indeed, the influence of the solid volume fraction on the viscosity is examined by identifying the maximum packing fraction and its value is here interpreted in the light of the DLVO theory. On the other hand, to the authors' knowledge, the rheological classification proposed by Coussot and Ancey [17] has never been achieved to the specific case of alumina suspensions. For the first time, this dimensionless approach is applied here in order to quantitatively identify the different flow regimes. It thus gives a new insight to discuss the rheology of alumina suspensions in light of their use in ceramic processing. In particular, this approach can help to choose a convenient operating range regarding the ceramic process which is considered.

\section{Material and methods}

\subsection{Alumina powder and dispersant}

A commercially available alumina powder (AKP-50, Sumitomo Chemical Co. Ltd., Japan) was used in this investigation. The median particle diameter of this high purity (>99.99\%) $\alpha$-alumina powder was found to be $206 \pm 4 \mathrm{~nm}$ using Zetasizer Nano ZS (Malvern Panalytical Ltd., UK). In addition, the specific surface area was found to be 11.50 $\pm 0.05 \mathrm{~m}^{2} . \mathrm{g}^{-1}$ using Tristar II 3020 (Micromeritics, USA).

An ammonium polymethacrylate (APMA) solution (Darvan ${ }^{\circledR} \mathrm{C}-\mathrm{N}$, Vanderbilt Minerals LLC, USA) was used as dispersant agent. It has an average molecular weight of about 13,000 g.mol ${ }^{-1}$ and it was supplied in the form of a $25 \mathrm{wt} \%$ aqueous solution. This organic compound is adsorbed onto the alumina surface because of hydrogen bondings and chemical interactions [24]. In addition, different conformations could be involved in this adsorption [25].

\subsection{Suspensions preparation}

Suspensions with various solid volume fractions of $\alpha-\mathrm{Al}_{2} \mathrm{O}_{3}$ powder were prepared according to the same procedure. First, the desired amount of APMA, expressed as a percentage of the alumina dry weight, is mixed with distilled water. The alumina powder is then progressively added to this water-based solution. In order to deagglomerate the powder, an ultrasonic treatment is applied using a probe (pulsed $3 \mathrm{~s}$ on $-1 \mathrm{~s}$ off, 1 min, 750 W, Vibracell ultrasonic desintegrator VC 750, Sonics \& Materials Inc., USA). Finally, until its characterization, each suspension is stored on a low-speed roller shaker.

\subsection{Zeta potential measurements}

The zeta potential of alumina particles was measured on diluted suspensions ( 1 vol\% of solid) as a function of $\mathrm{pH}$ using an ESA analyser (Acoustosizer IIs, Colloidal Dynamics LLC, USA). It was calculated from the ESA voltage data treated with the Acoustosizer IIs operation software by using the O'Brien relationship [26]. In order to cover a wide range of $\mathrm{pH}$ values (from 2 to 11), two suspensions were prepared for each slurry composition (without additives or with the optimum concentration of APMA). One was used to determine the zeta potential from natural to acidic $\mathrm{pH}$ while the other one was used for basic $\mathrm{pH}$. The suspension $\mathrm{pH}$ was adjusted using analytical grade of $\mathrm{HCl}$ and $\mathrm{NaOH}$ solutions $\left(0.1 \mathrm{~mol} . \mathrm{I}^{-1}\right)$. Moreover, for the suspensions without additives, the ionic strength was maintained using $\mathrm{NaCl}$ solution $\left(0.01 \mathrm{mol.1}^{-1}\right)$.

\subsection{Rheological behavior}

Rheological measurements were performed on dispersed suspensions with various solid volume fractions (from 1 vol\% to $40 \mathrm{vol} \%$ ) using an AR-G2 rheometer (TA Instruments, USA) with a cone-plane geometry ( $60 \mathrm{~mm}$ diameter, $53 \mu \mathrm{m}$ gap and $1^{\circ} 59^{\prime} 50^{\prime \prime}$ angle). This configuration was used because the shear rate is uniform in the whole sample volume. Moreover, the rheometer characteristics indicate a minimum measurable torque of $0.01 \mu \mathrm{N} . \mathrm{m}^{-1}$ and a minimum applicable shear rate of $2 \times 10^{-8} \mathrm{~s}^{-1}$. In order to give the same history to each sample and so get repeatable and consistent data, preliminary tests have been achieved on a dispersed suspension containing $25 \mathrm{vol} \%$ of $\mathrm{Al}_{2} \mathrm{O}_{3}$ particles to determine the required conditioning. Therefore, before each test, a preshear of $10 \mathrm{~s}^{-1}$ was applied for $30 \mathrm{~s}$ and was followed by 10 min at $0.01 \mathrm{~s}^{-1}$. Moreover, the rheological properties were measured following a logarithmic increase of the shear rate (from $0.01 \mathrm{~s}^{-1}$ to $200 \mathrm{~s}^{-1}$ ). Each measurement was achieved when the steady state was reached, i.e. the shear stress did not vary by more than $2 \%$ during $15 \mathrm{~s}$. If the maximum duration time of a step (120 s) was exceeded, the point was not retained and the strain rate was then increased up to the following measurement. Finally, all experiments were carried out at $20^{\circ} \mathrm{C}$.

\section{Results and discussion}

\subsection{Optimum concentration of dispersant}

First, in order to obtain a well dispersed suspension, its stability should be examined. In this respect, the evolution of the zeta potential of alumina suspensions as a function of $\mathrm{pH}$ is reported in Fig. 1.

Without additives, the isoelectric point (IEP) of the alumina suspension is measured at $\mathrm{pH}=9.5$. Furthermore, at the natural $\mathrm{pH}(\mathrm{pH}=$ 8.3 ), the zeta potential is assessed at $+21.7 \mathrm{mV}$. It is not high enough in absolute value to ensure a good dispersion of the particles in the suspension. Moreover, extreme $\mathrm{pH}$ values would be required to disperse the suspension. Consequently, the use of a dispersant agent is required. In order to determine the optimum concentration of this additive, rheological measurements were performed on 25 vol\% alumina 


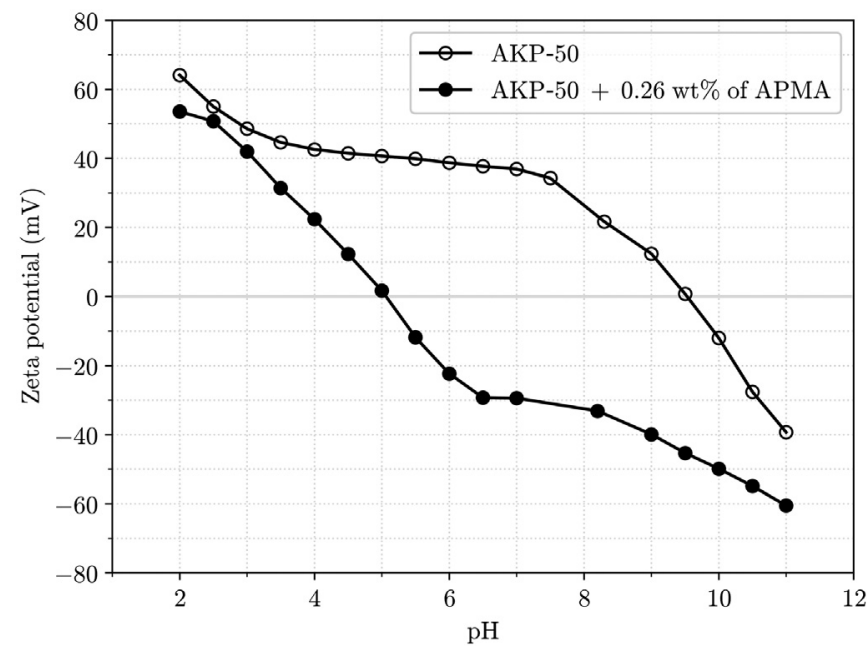

Fig. 1. Evolution of the zeta potential of alumina suspensions without additives and with $0.26 \mathrm{wt} \%$ of ammonium polymethacrylate as a function of the $\mathrm{pH}$.

suspensions with various amounts of ammonium polymethacrylate (Fig. 2). In fact, there is a critical concentration of dispersant which corresponds to a minimum of viscosity and to the best state of dispersion [27].

For small additions of dispersant, the viscosity of the suspension decreases. However, the quantity of dispersant is not high enough to reach the saturation of the surface of each particle by adsorbtion. When 0.26 wt\% of APMA is added, the viscosity reaches its minimum value and the stability is maximized. All the alumina particles are covered with ammonium polymethacrylate. If the dispersant continues to be added to the suspension, a part of it is no longer adsorbed onto particles and remains in the interstitial fluid, then increasing the ionic strength and compressing the electrostatic double layer. Thus, the viscosity of the suspension increases.

In order to confirm this optimum concentration of dispersant, zeta potential measurements were performed as well as particle size analysis. First, the evolution of the zeta potential of an alumina suspension containing $0.26 \mathrm{wt} \%$ of APMA as a function of the $\mathrm{pH}$ (Fig. 1) shows that the isoelectric point is shifted from $\mathrm{pH}=9.5$ (without additives) down to $\mathrm{pH}=5.1$. In consequence, at the natural $\mathrm{pH}(\mathrm{pH}=8.2)$, which does not vary, the zeta potential is now equal to $-33.1 \mathrm{mV}$

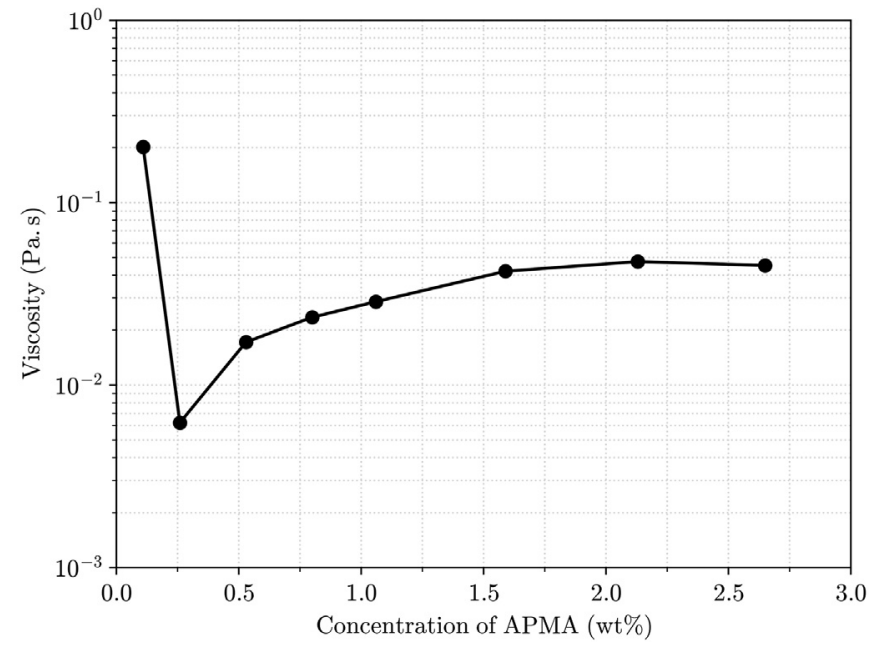

Fig. 2. Evolution of the viscosity of alumina suspensions ( $25 \mathrm{vol} \%$ ) at $200 \mathrm{~s}^{-1}$ as a function of the concentration of ammonium polymethacrylate. which ensures a good stability. The particle size distributions of alumina suspensions with and without dispersant are represented on Fig. 3.

Without dispersant, three populations of particles can be distinguished: the most important extends around $1 \mu \mathrm{m}$ and the larger diameter population exists around $10 \mu \mathrm{m}$, attesting the presence of agglomerates. Whereas, with 0.26 wt\% of APMA, the particle size distribution of the suspension presents a unique peak corresponding to the size of the ultrafine alumina powder (i.e. $0.2 \mu \mathrm{m}$ ). In conclusion, the optimum concentration of ammonium polymethacrylate required to formulate a well dispersed alumina suspension is $0.26 \mathrm{wt} \%$. Since then and later in this article, the term "suspension" refers to the suspension comprising the distilled water, the alumina powder and this amount of ammonium polymethacrylate.

Furthermore, the order of magnitude of the thickness of ammonium polymethacrylate adsorbed onto the surface of alumina particles can be estimated. On the one hand, if the dispersant is uniformly adsorbed onto the surface of particles, a lower bound of the thickness $t_{d}$ of APMA $\left(\rho^{\prime}=\right.$ $\left.1.11 \mathrm{~g} . \mathrm{cm}^{-3}\right)$, covering an alumina particle $(r=103 \mathrm{~nm}$ and $\rho=3.98 \mathrm{~g}$. $\mathrm{cm}^{-3}$ ) such as the relative weight percentage $\frac{\mathrm{m} \prime}{\mathrm{m}}$ is equal to $0.26 \mathrm{wt} \%$, corresponds to:

$\frac{m^{\prime}}{m}=\frac{\rho^{\prime} \cdot\left(\frac{4}{3} \pi\left(r+t_{d}\right)^{3}-\frac{4}{3} \pi r^{3}\right)}{\rho \cdot \frac{4}{3} \pi r^{3}} \Rightarrow t_{d}=r \cdot\left(\sqrt[3]{\frac{m^{\prime}}{m} \frac{\rho}{\rho^{\prime}}+1}-1\right)$

In this case, the value of $t_{d}$ is $0.32 \mathrm{~nm}$. However, adsorbed polymers are likely to be swollen or collapsed and their apparent density may differ from the bulk one. On the other hand, the thickness can also be evaluated according to De Gennes [28] assuming linear, flexible, neutral chains in good solvents:

$t_{d} \sim S . N^{\frac{3}{5}}$

with $s$ the monomer size $(s=0.27 \mathrm{~nm})$ and $N$ the number of monomers per chain $(N=126)$. According to this analytical formula, $t_{d}$ is equal to $4.85 \mathrm{~nm}$. Both methods provide extreme values corresponding to the various conformations of the APMA adsorbed onto the alumina surface [25]. Assuming that, in this case, it is an intermediate configuration, it can be considered that the thickness of the layer is of the order of one nanometer.

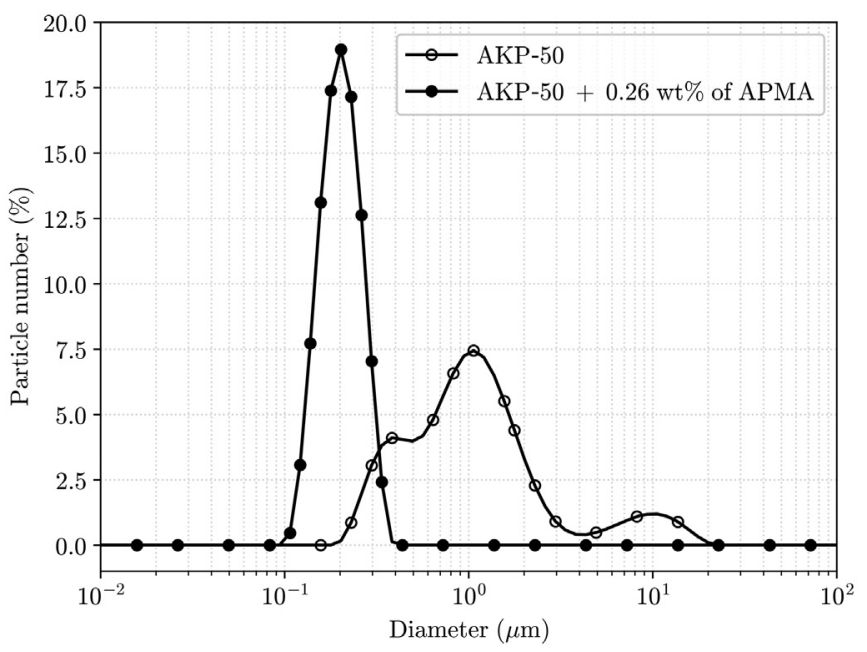

Fig. 3. Particle size distributions of alumina suspensions without additives or with $0.26 \mathrm{wt}$ $\%$ of ammonium polymethacrylate (Mastersizer 3000, Malvern Panalytical Ltd., UK). 
(a)

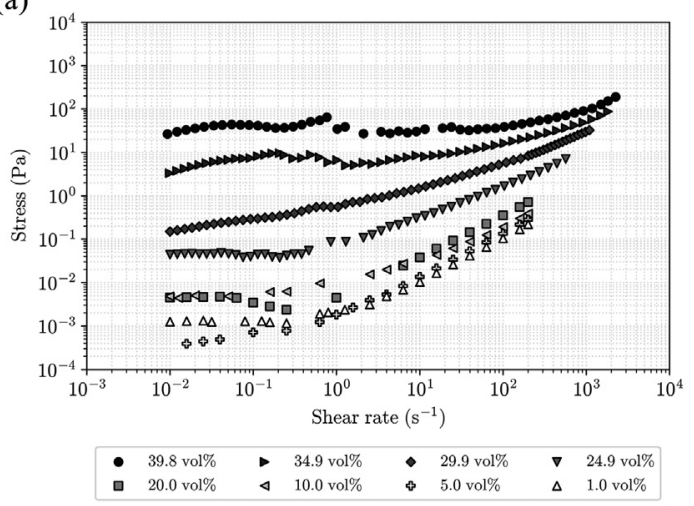

(b)

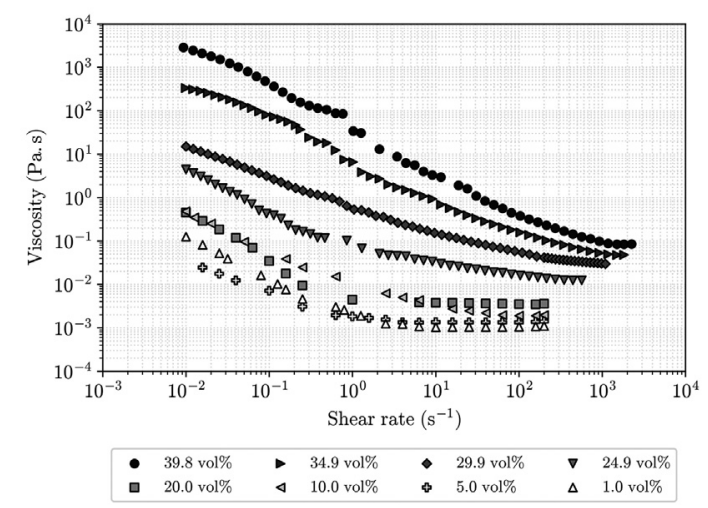

Fig. 4. Rheograms of dispersed alumina suspensions for various solid volume fractions (from 1 vol\% to 39.8 vol\%) - (a) Stress vs Shear rate - (b) Viscosity vs Shear rate.

\subsection{Rheological behavior of dispersed alumina suspensions}

Fig. 4 represents the rheograms of dispersed alumina suspensions for various solid volume fractions.

Regardless of the solid volume fraction, the rheological behavior of a suspension could be divided into two domains (Fig. 4b). First, at low shear rates, the viscosity decreases. In other words, the suspension exhibits a shear thinning behavior. Then, it is followed, at high shear rates, by a constant viscosity plateau. In order to evidence the plateau for concentrated suspensions, the measurements were performed beyond $200 \mathrm{~s}^{-1}$. This behavior is due to the destructuration of the suspension. Because of the interparticle interactions, the suspension initially presents a certain structure which is responsible for flow resistance. When the shear rate increases, the energy brought by the flow progressively breaks the interparticle interactions. It macroscopically involves the existence of a yield stress and a decrease of the viscosity. Above a given shear rate, the suspension no longer has time to restore its structure. Thus, only hydrodynamic interactions predominate. In other words, the viscosity remains constant.

In agreement with the classification of Coussot and Ancey [17], the rheological behavior of dispersed alumina suspensions is here dictated by three main types of interactions for low-to-medium concentrations and shear rates: Brownian motions, colloidal interactions and hydrodynamic interactions. Those physical phenomena are present simultaneously in a suspension even if one of them predominates over others mainly depending on the solid volume fraction, on the interactions between particles and on the shear rate.

First, the interstitial fluid possesses a Newtonian behavior. When a particle is added, the velocity field is modified. In fact, the fluid has to flow around the solid element. In consequence, a drag force, as expressed by the Stokes' law, exists. The behavior is still Newtonian but the viscosity of the suspension is higher because of the excess of hydrodynamic energy resulting from this perturbation. Moreover, when the solid volume fraction increases, each particle perturbates the velocity field of every other particle. In other words, in this regime, the particles interact in a hydrodynamic way. In addition, this implies that the higher the solid volume fraction, the higher the viscosity. In Fig. 4b, the hydrodynamic domain can be identified as the plateau, with a constant viscosity, higher than the one of water and which increases with the solid volume fraction. Finally, this domain appears from $1 \mathrm{~s}^{-1}$ for low solid volume fractions but it could begin beyond $10^{3} \mathrm{~s}^{-1}$ for the higher solid volume fractions.

Furthermore, Brownian motions and colloidal interactions are responsible for the shear thinning behavior of the suspensions. On the one hand, the molecules of the interstitial fluid bump the particles of the suspension because of the thermal kinetic energy. Thus, the particles move away from their initial positions and have random movements which are independent from the macroscopic flow imposed to the suspension. These Brownian movements are particularly important when the particles are smaller than a few microns [29], like here. In addition, the viscosity of suspensions in this domain is higher than the one in the hydrodynamic regime. Indeed, Brownian movements induce a diffusion of the particles which leads to momentum transfers. Thus, to maintain a constant shear in the suspension, it is necessary to increase the tangential force. This induces additional hydrodynamic dissipations and therefore higher viscosity.

On the other hand, distance-dependent interactions exist between particles due to colloidal forces. They include Van der Waals forces, electrostatic forces and those related to steric effects due to adsorption of polymers onto the particle surface. They can be either repulsive or attractive and the higher the solid volume fraction, the higher the total sum of interactions in the system. In consequence, there exists an equilibrium structure for which each particle is embedded within a network. In order to impose a homogeneous flow on the suspension, this network must be broken and each particle has to be extracted from its equilibrium position. Macroscopically, an apparent yield stress should be overcome. So the viscosity is higher in this domain than in the hydrodynamic one.

As a consequence, the Brownian regime corresponds to the shear thinning domain of suspensions with low solid volume fractions whereas the colloidal regime characterizes suspensions with high solid volume fractions. There is a regime transition for a concentration comprised between $20 \mathrm{vol} \%$ and $24.9 \mathrm{vol} \%$. Below this solid volume fraction, the flow curves show no order because of Brownian motions. Moreover, fewer data were recorded in this region because it was difficult to reach the steady state during the measurement. Nonetheless, such an order exists for the suspensions dominated by colloidal interactions. In addition, the transitions between those regimes and the hydrodynamic one are consistent with this identification. Indeed, the energy, and thus the shear rate required to overcome Brownian motions, are lower than the ones in the case of colloidal interactions.

In brief, at low shear rates, the behavior of diluted suspensions is mainly governed by Brownian motions whereas for concentrated ones, colloidal interactions dominate. In both cases, increasing the shear rate promotes the predominance of hydrodynamic interactions.

\subsection{Maximum packing fraction}

Following the description of the rheological behavior of dispersed alumina suspensions (Section 3.2), it can be confirmed that solid volume fraction is an important property which defines a suspension. In order to study the effect of this characteristic on viscosity, the Maron and Pierce model was used to evaluate the maximum packing fraction:

$\sqrt{\frac{\eta_{0}}{\eta}}=1-\frac{\varphi}{\varphi_{\max }}$ 
where $\eta$ is the viscosity of the suspension, $\eta_{0}$ the viscosity of the interstitial fluid, $\varphi$ the solid volume fraction of the suspension and $\varphi_{\max }$ the maximum packing fraction. On the one hand, the hydrodynamic domain, corresponding to the plateau at which the viscosity is constant, is the only regime common to all suspensions regardless of the solid volume fraction and then permits to compare suspensions which are in the same condition. Thus, for each suspension, it is possible to associate a unique value of viscosity. On the other hand, using the optimum concentration of dispersant, it can be assumed that the entire amount of ammonium polymethacrylate is adsorbed onto the surface of alumina particles and that there are no residues in water. The viscosity of the interstitial fluid is constant and equal to the water viscosity ( 1 mPa.s). In consequence, $\varphi_{\max }$ is determined by calculating the slope of the linear fit of the data (Fig. 5).

The data fit is quite good $\left(\mathrm{R}^{2}=0.976\right)$. The maximum packing fraction of dispersed alumina suspensions was found to be $0.40 \pm 0.03$ (confidence interval for a confidence level of 95\%). This value is close to the highest solid volume fraction examined in this study (i.e. 0.398). However, the data fit seems to be relatively weak for suspensions with a solid volume fraction close to the maximum packing fraction. It suggest that the true value of the maximum packing fraction could be slightly higher than 0.40 . Nonetheless, this value was considered in the rest of the article. Furthermore, it is consistent with the literature according to the particle size (i.e. $200 \mathrm{~nm}$ ). Using a Maron and Pierce model, a maximum packing fraction of $43.1 \mathrm{vol} \%$ was determined by Billote et al. [16] for alumina suspensions $\left(d_{50}=0.3 \mu \mathrm{m}\right)$ with nitric acid as dispersant and PVA as binder. Moreover, the value identified in the present work can be compared to a theoretical one. According to Buscall et al. [21], at low shear rates, the theoretical maximum packing fraction of monosized spherical particles is equal to 0.64 whereas, at high shear rates, its value is 0.74 . Given the fact that $\varphi_{\max }$ was identified from viscosity values in the hydrodynamic regime, the second value is taken as reference. The gap between the measured and theoretical values can be interpreted by the fact that particles are kept at a certain distance from each other even if the solid volume fraction of the suspension is maximum. Unlike the theoretical configuration based on hard spheres, the particle surfaces are not in direct contact here. So it amounts to consider that there is a layer homogeneously distributed around each particle and it is the outer envelopes of these layers that are in contact. The existence of a layer of thickness $t$ around each particle of radius $r$ leads to a discrepancy between the measured and theoretical values of $\varphi_{\max }$ that can be expressed as:

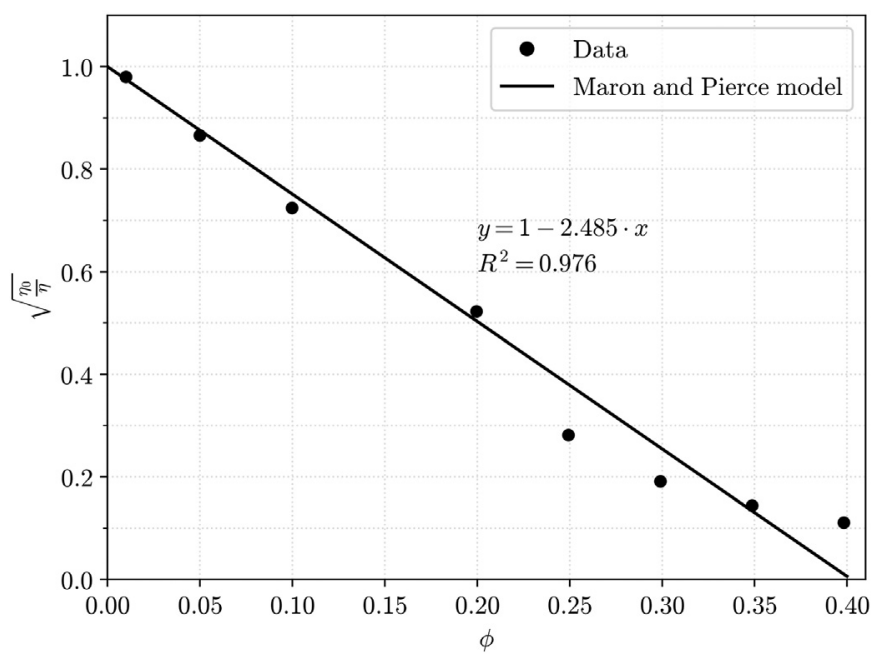

Fig. 5. Identification of the maximum packing fraction by linear regression according to the Maron and Pierce model.

$$
\frac{\varphi_{\max }^{\text {measure }}}{\varphi_{\max }^{\text {theory }}}=\frac{r^{3}}{(r+t)^{3}} \Rightarrow t=r \cdot\left[\frac{1}{\sqrt[3]{\frac{\varphi_{\text {meax }}^{\text {meare }}}{\varphi_{\max }^{\text {theory }}}}}-1\right]
$$

For dispersed alumina suspensions, $t$ is evaluated at $23 \pm 3 \mathrm{~nm}$. This value is significantly higher than the one of the thickness of ammonium polymethacrylate adsorbed onto the alumina surface (i.e. $\approx 1 \mathrm{~nm}$ ). In consequence, the steric effect alone cannot explain the separation distance between particles. However, even if the maximum packing fraction was identified in the hydrodynamic domain, colloidal interactions exist and can have an effect on the arrangement of the particles. In order to estimate the intensity of Van der Waals and electrical double layer forces, the DLVO theory is applied.

On the one hand, the attractive Van der Waals interaction potential of two identical spherical particles of radius $r(r=103 \mathrm{~nm})$ separated by a distance $b$ between the center of both particles is approximately given by [30]:

$V_{A}=\frac{-A}{6}\left[\frac{2 r^{2}}{b^{2}-4 r^{2}}+\frac{2 r^{2}}{b^{2}}+\ln \left(1-\frac{4 r^{2}}{b^{2}}\right)\right]$

with $A$ the Hamaker constant. For two alumina particles dispersed in water, $A$ is equal to $4.76 \times 10^{-20} \mathrm{~J}$ [31].

On the other hand, the repulsive electrical double layer potential can be approximately expressed by [6]:

$V_{R}=2 \pi \varepsilon_{r} \varepsilon_{0} r \Phi_{d}^{2} \cdot e^{-\kappa(b-2 r)}$

where $\varepsilon_{r}$ is the relative permittivity of water $\left(\varepsilon_{r}=81\right.$ [31]), $\varepsilon_{0}$ the vacuum permittivity $\left(\varepsilon_{0}=8.85 \times 10^{-12} \mathrm{~F} . \mathrm{m}^{-1}\right)$ and $\Phi_{d}$ the diffuse layer potential which is typically equivalent to the zeta potential. According to the electrophoretic mobility measurements, $\zeta$ is equal to $-33.1 \mathrm{mV}$ at natural $\mathrm{pH}$ in presence of $0.26 \mathrm{wt} \%$ of ammonium polymethacrylate. The Debye length $\kappa^{-1}$ is given by:

$\kappa^{-1}=\left(\frac{\varepsilon_{r} \varepsilon_{0} k T}{2 N_{A} e^{2} I}\right)^{\frac{1}{2}}$

where $k$ is the Bolztmann constant $\left(k=1.38 \times 10^{-23} J . \mathrm{K}^{-1}\right), T$ the temperature $(T=293 \mathrm{~K}), N_{A}$ the Avogadro constant $\left(N_{A}=\right.$ $\left.6.02 \times 10^{-23} \mathrm{~mol}^{-1}\right)$ and $e$ the elementary charge $\left(e=1.06 \times 10^{-19} \mathrm{C}\right)$. The ionic strength $I$ can be estimated from the electrical conductivity $\sigma$ of the suspensive water determined during the zeta potential measurements $\left(\sigma=71.0 \mu \mathrm{S} . \mathrm{cm}^{-1}\right)$ [32]:

$I=0.013 . \sigma$

if $I$ is expressed in mol.m ${ }^{-3}$ and $\sigma$ in $\mu \mathrm{S} . \mathrm{cm}^{-1}$. Thus the Debye length was found to be $10.1 \mathrm{~nm}$.

The total potential $V_{T}$ is equal to the sum of attractive $V_{A}$ and repulsive $V_{R}$ potentials. The evolutions of these potentials are plotted in Fig. 6a while the derivative of $V_{T}$ is represented on Fig. $6 \mathrm{~b}$.

The profile of $V_{T}$ shows a maximum value of $66 k T$ for $b-2 r=3.2$ $\mathrm{nm}$. Moreover, the total potential is zero for $b-2 r=63.8 \mathrm{~nm}$ and a secondary minimum $\left(-9 \times 10^{-2} k T\right)$ exists for $b-2 r=81.2 \mathrm{~nm}$. The colloidal interactions, which are, in this case, mainly predominated by the repulsive forces to ensure a dispersed state, will maintain the particles at a certain distance from each other. Considering that those forces are negligible when the total potential reaches $1 \%$ of the energy barrier $\left(66 \times 10^{-2} k T\right)$, it is possible to estimate the minimum distance between two alumina particles $(47.9 \mathrm{~nm})$. This value is similar to twice the thickness $t$ estimated from the identification of the maximum packing fraction $(2 t=46 \mathrm{~nm})$.

Although this approach considers average values and large fluctuations may occur in the system, this still suggests that, when the solid 

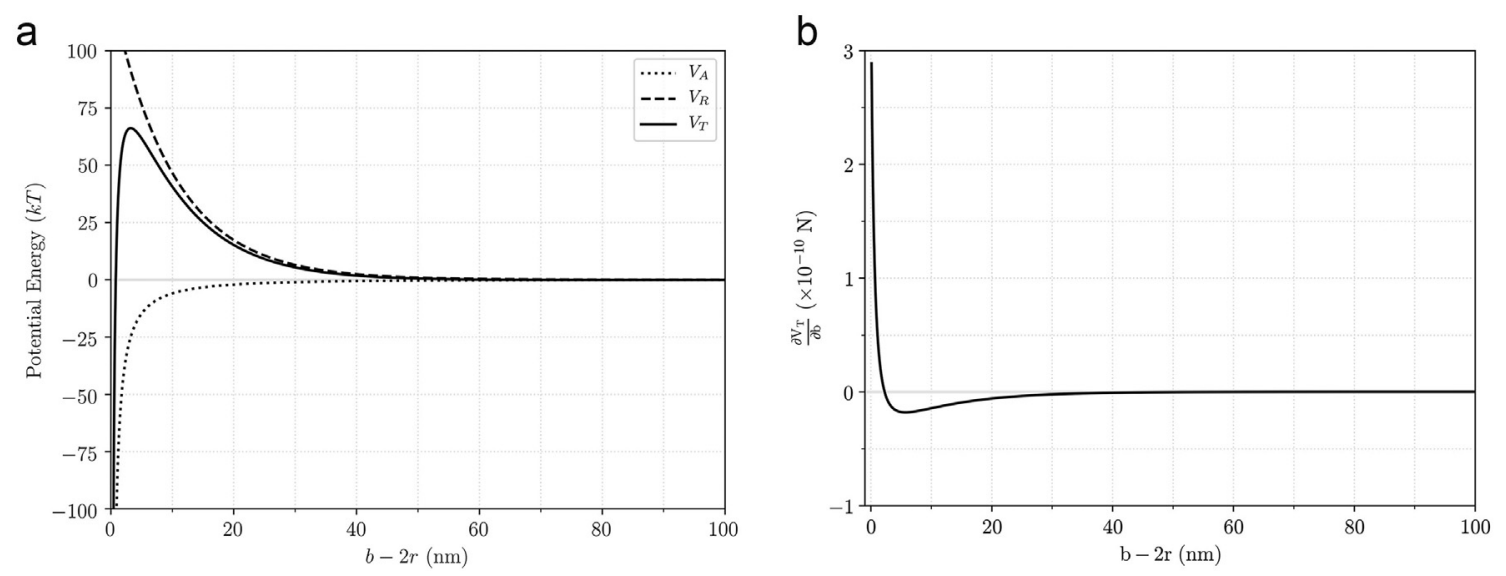

Fig. 6. Application of the DLVO theory to alumina suspensions - (a) Evolution of $V_{A}, V_{R}$ and $V_{T}$ as a function of b-2r - (b) Evolution of the derivative of $V_{T}$ as a function of b-2r.

volume fraction of the suspension is maximum, the particles cannot be in contact because of colloidal interactions. The separation distance thus seems to correspond to the one at which those interactions become negligible. In conclusion, at a macroscopic scale, the maximum packing fraction of a suspension reflects the intensity of colloidal interactions occurring at a microscopic scale.

\subsection{Rheophysical classification of dispersed alumina suspensions}

According to Section 3.2, the rheological behavior of dispersed alumina suspensions is dictated by three main types of interactions. The different flow regimes associated to each predominant interaction were qualitatively identified. The aim of this final section is to apply the rheophysical classification proposed by Coussot and Ancey [17] in order to allow a quantitative identification. The objective is to draw the different frontiers on the rheogram of alumina suspensions (Fig. 4b).

First, colloidal interactions and Brownian motions can be compared using the dimensionless number $N_{r}$ expressed by [17]:

$N_{r}=\frac{\psi_{0}}{k T}$

where $\psi_{0}$ is an energy barrier which characterizes the strength of the interparticle interactions and $k T$ the typical energy associated to thermal agitation ( $k$ is the Boltzmann constant and $T$ the temperature). When $N_{r}$ is largely greater than 1 , Brownian motions are negligible compared to colloidal interactions and conversely if $N_{r} \ll 1$. It means that the curve $N_{r}=1$ approximately corresponds to the transition between a regime for which Brownian motions dominate toward a regime for which colloidal interactions dominate.

Statistical mechanics [33] provides a general expression for the shear modulus of suspensions, in which two quantities appear. The first one corresponds to the typical energy associated to thermal agitation whereas the second one, involving the pair interaction potential and a particle distribution function, reflects the contribution of the environment. By assimilating this last quantity to the energy barrier, a simplified expression of $\psi_{0}$ is [34]:

$\left.\psi_{0}=\frac{q \varphi_{\max }}{5 \pi b n} \cdot \frac{4}{b} \cdot \frac{\partial V_{T}(b)}{\partial b}+\frac{\partial^{2} V_{T}(b)}{\partial^{2} b}\right)$

where $q$ is the corresponding number of nearest neighbors, $\varphi_{\max }$ the maximum packing fraction, $b$ the characteristic mean distance between the centers of two neighboring particles, $n$ the particle number density and $V_{T}(b)$ the total pair interaction potential.

When the maximum packing fraction is reached, it is assumed that the arrangement taken by the particles is face-centered cubic.
Moreover, it is supposed that this arrangement is kept in an "expansed" form for lower solid volume fractions. In consequence, the value of $q$ is equal to 12 . Furthermore, $n$ and $b$ depend on the solid volume fraction $\varphi$ :

$n=\frac{\varphi}{\frac{4}{3} \pi r^{3}}$

$b=2 \cdot(r+t) \cdot\left(\frac{\varphi}{\varphi_{\max }}\right)^{\frac{-1}{3}}$

In the case of dispersed alumina suspensions, it was shown (see Section 3.3) that the colloidal interactions prevent direct contact between particles. This separation is associated to a layer thickness $t$ around each particle $(t=23 \mathrm{~nm})$. Thus, in the last expression (Eq. 14), the distance between the centers of particles is equal to 2 . $(r+t)$ when $\varphi=\varphi_{\max }$. Moreover, the distance $b$ will increase when the solid volume fraction decreases. Finally, the total potential $V_{T}(b)$ has been calculated in Section 3.3 for alumina suspensions thanks to the DLVO theory.

In consequence, the barrier energy $\psi_{0}$ can be defined as a function of the solid volume fraction. Thus, it is possible to identify the solid volume fraction $\varphi_{N r=1}$ associated to $N_{r}=1$ i.e. $\psi_{0}=k T$. For studied dispersed alumina suspensions, it is equal to 0.233 . This value is consistent with the one qualitatively identified in Section 3.2 since it is between 20.0 vol\% and 24.9 vol\%.

Nonetheless, in order to draw this transition on the rheogram, it is necessary to represent the evolution of the viscosity as a function of the shear rate for this suspension containing $23.3 \mathrm{vol} \%$ of alumina. The Bingham model [35], for which the stress is the sum of a yielding term and a Newtonian-like term, is often used in literature to described a yield-flow behavior. It is expressed as:

$\tau=\tau_{0}+\eta_{p} \dot{\gamma}$

where $\tau$ is the shear stress, $\dot{\gamma}$ the shear rate, $\tau_{0}$ a yield stress and $\eta_{p}$ a plastic viscosity. On the one hand, the energy barrier $\psi_{0}$ implies the existence of a yield stress. The following equation provides a basic relation between these two quantities [36]:

$\tau_{0}=(0.016+0.52 \varphi) \cdot \psi_{0} \cdot b^{-3}$

On the other hand, the plastic viscosity can be assimilated to the viscosity of the suspension in the hydrodynamic domain. Thus, it can be estimated thanks to the Maron and Pierce model. Finally, the transition between the regime for which Brownian motions dominate toward 
the regime for which colloidal interactions dominate can be represented on the rheogram by:

$\eta=\frac{\left(0.016+0.52 \varphi_{N_{r}=1}\right) \cdot k T \cdot \frac{\varphi_{N_{r}=1}}{\varphi_{\max }}}{8 \dot{\gamma}(r+t)^{3}}+\eta_{0} \cdot\left(1-\frac{\varphi_{N_{r}=1}}{\varphi_{\max }}\right)^{-2}$

At low shear rates, the rheological behavior of alumina suspensions with solid volume fractions lower than 0.233 is governed by Brownian motions. Increasing the shear rate promotes the predominance of hydrodynamic interactions. The Péclet number $P e$ is a dimensionless number defined as the ratio of hydrodynamic dissipations with the thermal energy [17]:

$P e=\frac{K \mu b^{2} \dot{\gamma}}{k T}$

$K$ is a coefficient which depends on the shape, the size and the orientation of the particles. For a sphere, $K$ is equal to $6 \pi r$. $\mu$ can also be assimilated to the viscosity of the interstitial fluid $\eta_{0}$. It is important to underline the fact that the influence of the solid volume fraction can be expressed through the definition of $b$ (Eq. 14). Brownian motions are predominant during flow when this dimensionless number is largely smaller than 1 whereas the flow regime is dominated by the hydrodynamic interactions when $P e \gg 1$. The curve $P e=1$ thus corresponds to the transition between a regime for which Brownian motions dominate toward a regime for which hydrodynamic interactions dominate. Moreover, for a suspension with a given solid volume fraction, it is possible to express the shear rate corresponding to this frontier:

$\dot{\gamma}_{P e=1}=\frac{k T}{6 \pi r \eta_{0} b^{2}}$

However, in order to represent this transition on the rheogram of alumina suspension, it is necessary to associate a viscosity $\eta_{P e=1}$ to this shear rate $\dot{\gamma}_{P e=1}$. Given the flow regimes on either side of this limit, the viscosity $\eta_{P e=1}$ could be assimilated to the one of the plateau which can be expressed thanks to the Maron and Pierce model provided that the transition line actually intersects the plateau of each curve, which is checked a posteriori (Fig. 7). Finally, the transition between the regime for which Brownian motions dominate toward the regime for which hydrodynamic interactions dominate corresponds to the parametric curve defined by:

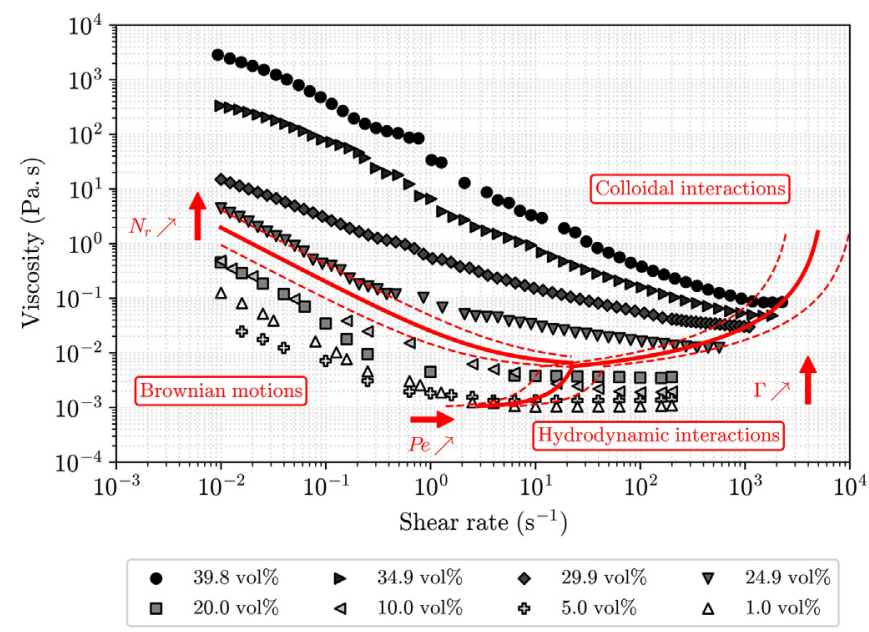

Fig. 7. Identification of the flow regimes on the rheogram of dispersed alumina suspensions. The continuous lines correspond to dimensionless numbers equal to 1 whereas dotted lines represent dimensionless numbers of $1 / 2$ and 2 .

$$
\left.\dot{\gamma}_{P e=1}=\frac{k T}{6 \pi r \eta_{0} b^{2}} ; \eta_{P e=1}=\eta_{0} \cdot\left(1-\frac{\varphi}{\varphi_{\max }}\right)^{-2}\right)
$$

On the other hand, if the solid volume fraction of a suspension is greater than 0.233 , its rheological behavior is dominated by colloidal interactions at low shear rates. Increasing the shear rate promotes the predominance of hydrodynamic interactions. In order to estimate the importance of colloidal interactions compared to hydrodynamic interactions, the dimensionless number $\Gamma$ can be defined [17]:

$\Gamma=\frac{K \mu b^{2} \dot{\gamma}}{\psi_{0}}$

Hydrodynamic interactions are predominant during flow when this ratio is largely greater than 1 whereas the flow regime is dominated by colloidal interactions when $\Gamma \ll 1$. The curve $\Gamma=1$ thus corresponds to the transition between a regime for which colloidal interactions dominate toward a regime for which hydrodynamic interactions dominate. It is also possible, for a suspension with a given solid volume fraction, to express the shear rate corresponding to this limit:

$\dot{\gamma}_{\Gamma=1}=\frac{\psi_{0}}{6 \pi r \eta_{0} b^{2}}$

with $\psi_{0}$ the barrier energy as expressed in Eq. 12. As exploited previously, the viscosity $\eta_{\Gamma=1}$ associated to this shear rate can be estimated thanks to the Maron and Pierce model provided that the transition line actually intersects the plateau of each curve, which is checked a posteriori (Fig. 7). Finally, the transition between the regime for which colloidal interactions dominate toward the regime for which hydrodynamic interactions dominate corresponds to the parametric curve defined by:

$$
\left.\dot{\gamma}_{\Gamma=1}=\frac{\psi_{0}}{6 \pi r \eta_{0} b^{2}} ; \eta_{\Gamma=1}=\eta_{0} \cdot\left(1-\frac{\varphi}{\varphi_{\max }}\right)^{-2}\right)
$$

Consequently, the equations Eq. 17, Eq. 20 and Eq. 23 allow to draw, on the rheogram of dispersed alumina suspensions, the frontiers between the different flow regimes (Fig. 7).

The plot of the various transitions accurately matches with the flow regimes as discussed in Section 3.2. For low solid volume fractions, the curves show no order due to the predominance of Brownian motions. When the solid volume fraction is sufficiently high, such an order appears. The viscosity of the suspensions increases significantly. Colloidal interactions predominate. Furthermore, in both cases, increasing shear rate promotes the predominance of hydrodynamic interactions. In this regime, the viscosity reaches a plateau. Additionally, it can be observed that the slope, which is defined by the Bingham model for the transition between the regime for which Brownian motions dominate toward the regime for which colloidal interactions dominate, is also valid for the suspensions with a solid volume fraction lower than 0.233 but it is not the case for the others governed by the colloidal interactions.

In the classification of Coussot and Ancey, the curves $N_{r}=1, P e=1$ and $\Gamma=1$ intersect in a single point for which Brownian, colloidal and hydrodynamic effects are more or less balanced. Here, this point exists for $\dot{\gamma}=22 \mathrm{~s}^{-1}$ and $\varphi=0.233$. The associated viscosity is $5 \times 10^{-3}$ Pa.s. Moreover, the solid volume fraction corresponding to $N_{r}=1$ is a concentration at which the intensity of colloidal interactions implies a major change in the rheological behavior. In fact, the shear rate corresponding to the beginning of the transition delimiting the hydrodynamic regime varies suddenly with the physical phenomenon that should take place. Since Brownian motions require less energy to become negligible, the shear rate at the boundary is low. On the other hand, colloidal interactions are stronger and thus require more energy, 


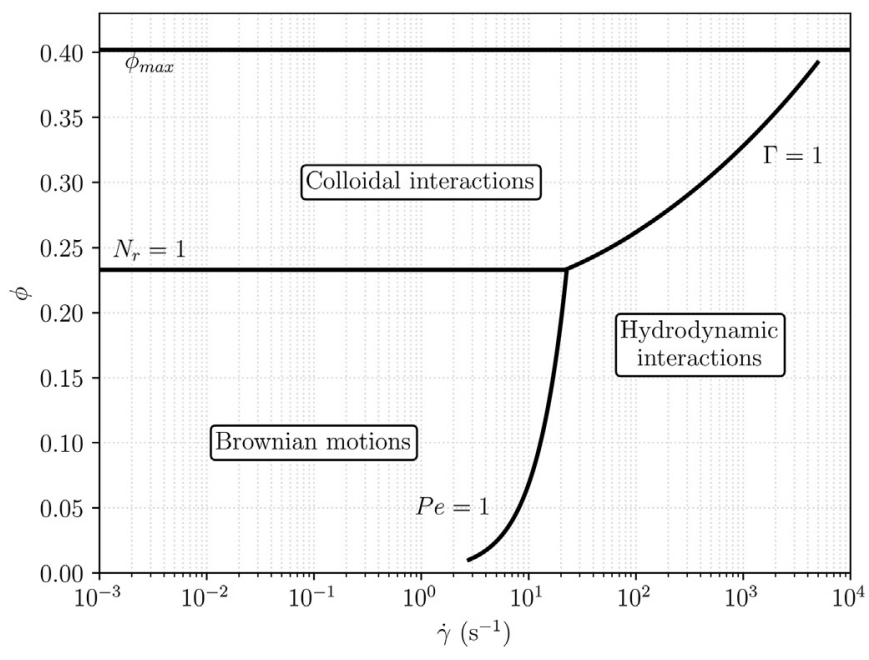

Fig. 8. Rheophysical classification of dispersed alumina suspensions.

which results in a higher shear rate. Furthermore, the transition curves, which have been drawn considering dimensionless numbers equal to 1 , do not correspond to an exact limit between two regimes, but simply point out a region around which it can be expected that the type of predominant interactions progressively changes. In this respect, lower and upper bonds are arbitrarily drawn in Fig. 7 by considering dimensionless numbers respectively equal to $1 / 2$ and 2 (dotted lines).

In conclusion, the dimensionless approach performed in this section makes it possible to identify the interactions type that dictates the rheological behavior of a suspension with a solid volume fraction $\varphi$ on which a shear rate $\dot{\gamma}$ is applied (Fig. 8). This approach is particularly interesting because it can help to choose a convenient operating range which is suitable for the intended ceramic process.

\section{Conclusions}

Understanding the rheological behavior of suspensions is required to obtain high quality products by ceramic colloidal processes. In this respect, a rheophysical study of aqueous fine alumina suspensions has been performed.

First, a good state of dispersion is satisfied by adding a concentration of $0.26 \mathrm{wt} \%$ of ammonium polymethacrylate.

Then, the evolution of the viscosity as a function of shear rate is divided into two domains: a shear thinning one followed by a plateau at which the viscosity is constant. This behavior is dictated by three main interaction types: Brownian motions, colloidal interactions and hydrodynamic interactions. All these interactions take place in the suspension but, depending on the shear rate and the solid volume fraction, one can be predominant over the others. At low shear rates, Brownian motions govern the behavior of suspensions with low solid volume fractions. Increasing the particles concentration increases the influence of colloidal interactions. In both cases, when the shear rate is high enough, hydrodynamic interactions are predominant.

In addition, the effect of solid volume fraction on viscosity was investigated by identifying the maximum packing fraction as defined in the Maron and Pierce model. By using the viscosity values in the hydrodynamic domain, this critical fraction was found to be $40 \mathrm{vol} \%$ for this AKP-50 alumina suspension dispersed with $0.26 \mathrm{wt} \%$ of ammonium polymethacrylate. The gap between this calculated value and the theoretical one was interpreted by the fact that the particles are maintained at a surface-to-surface separation distance of about $46 \mathrm{~nm}$. As described by the DLVO theory, this space corresponds to the minimum distance from which the repulsive potential is large enough. In consequence, a link between the rheology and the DLVO theory was established given the fact that the maximum packing fraction of a suspension reflects, at a macroscopic scale, the intensity of colloidal interactions occurring at a microscopic scale.

Finally, a dimensionless approach was achieved to quantitatively identify, on the rheogram, the different flow regimes associated to each predominant interaction type. This is particularly useful for the choice of a convenient operating range for the ceramic processing.

\section{Declaration of Competing Interest}

The authors declare that they have no known competing financial interests or personal relationships that could have appeared to influence the work reported in this paper.

\section{Acknowledgements}

This research received a grant from the Occitanie region and fundings from Institut Carnot M.I.N.E.S (ANR).

\section{References}

[1] J.A. Lewis, Colloidal processing of ceramics, J. Am. Ceram. Soc. 83 (2000) 2341-2359, https://doi.org/10.1111/j.1151-2916.2000.tb01560.x.

[2] R. Moreno, Colloidal processing of ceramics and composites, Adv. Appl. Ceram. 111 (2012) 246-253, https://doi.org/10.1179/1743676111Y.0000000075.

[3] R. Moreno, Better ceramics through colloid chemistry, J. Eur. Ceram. Soc. 40 (2020) 559-587, https://doi.org/10.1016/j.jeurceramsoc.2019.10.014.

[4] F.F. Lange, Powder processing science and technology for increased reliability, J. Am. Ceram. Soc. 72 (1989) 3-15, https://doi.org/10.1111/j.1151-2916.1989.tb05945.x.

[5] H.U. Kayaci, S. Cinar, Inter-particle spacing in aqueous suspensions of nanopowders and its effects on particle packing, green body formation and fabrication of alumina, Ceram. Int. 46 (2020) 20357-20368, https://doi.org/10.1016/j.ceramint.2020.05. 126.

[6] G.V. Franks, Y. Gan, Charging behavior at the alumina-water interface and implications for ceramic processing, J. Am. Ceram. Soc. 90 (2007) 3373-3388, https://doi. org/10.1111/j.1551-2916.2007.02013.x.

[7] B. Derjaguin, L. Landau, Theory of the stability of strongly charged lyophobic sols and of the adhesion of strongly charged particles in solutions of electrolytes, Acta Physicochim. URSS 14 (1941) 633-662, https://doi.org/10.1016/0079-6816(93) 90013-L.

[8] E.J.W. Verwey, J.T.G. Overbeek, K. van Nes, Theory of the stability of lyophobic colloids: the interaction of sol particles having an electric double layer, Elsevier Pub. Co., New York, 1948.

[9] B. Salopek, D. Krasic, S. Filipovic, Measurement and application of zeta-potential, Rud.-Geolosko-Naft. Zb. 4 (1992) 147-151.

[10] Z. Zhou, P.J. Scales, D.V. Boger, Chemical and physical control of the rheology of concentrated metal oxide suspensions, Chem. Eng. Sci. 56 (2001) 2901-2920, https:// doi.org/10.1016/S0009-2509(00)00473-5.

[11] A.I. Gómez-Merino, J.L. Arjona-Escudero, I.M. Santós-Ráez, F.J. Rubio-Hernández, Microstructure and thermodynamic properties of aqueous alumina nanofluids, Powder Technol. 353 (2019) 509-515, https://doi.org/10.1016/j.powtec.2019.05.054.

[12] R.C.D. Cruz, A.M. Segadães, P.Q. Mantas, R. Oberacker, M.J. Hoffmann, Interpreting rheology and electrical conductivity: it all boils down to which particle size, J. Colloid Interface Sci. 574 (2020) 97-109, https://doi.org/10.1016/j.jcis.2020.04.046.

[13] X. Xu, S. Zhou, J. Wu, C. Zhang, X. Liu, Inter-particle interactions of alumina powders in UV-curable suspensions for DLP stereolithography and its effect on rheology, solid loading, and self-leveling behavior, J. Eur. Ceram. Soc. 41 (2021) 2763-2774, https://doi.org/10.1016/j.jeurceramsoc.2020.12.004.

[14] A. Tsetsekou, C. Agrafiotis, A. Milias, Optimization of the rheological properties of alumina slurries for ceramic processing applications part I: slip-casting, J. Eur. Ceram. Soc. 21 (2001) 363-373, https://doi.org/10.1016/S0955-2219(00)00185-0.

[15] A. Tsetsekou, C. Agrafiotis, I. Leon, A. Milias, Optimization of the rheological properties of alumina slurries for ceramic processing applications part II: spray-drying, J. Eur. Ceram. Soc. 21 (2001) 493-506, https://doi.org/10.1016/S0955-2219(00) 00232-6.

[16] C. Billotte, E.R. Fotsing, E. Ruiz, Optimization of alumina slurry for oxide-oxide ceramic composites manufactured by injection molding, Adv. Mater. Sci. Eng. 2017 (2017) 1-9, https://doi.org/10.1155/2017/2748070.

[17] P. Coussot, C. Ancey, Rheophysical classification of concentrated suspensions and granular pastes, Phys. Rev. E 59 (1999) 4445-4457, https://doi.org/10.1103/ PhysRevE.59.4445.

[18] A. Einstein, Eine neue Bestimmung der Moleküldimensionen, Ann. Phys. 324 (1906) 289-306.

[19] A. Einstein, Berichtigung zu meiner Arbeit: Eine neue Bestimmung der Moleküldimensionen, Ann. Phys. 339 (1911) 591-592.

[20] S.H. Maron, P.E. Pierce, Application of ree-eyring generalized flow theory to suspensions of spherical particles, J. Colloid Sci. 11 (1956) 80-95, https://doi.org/10.1016/ 0095-8522(56)90023-X. 
[21] R. Buscall, P. D'Haene, J. Mewis, Maximum density for flow of dispersions of near monodisperse spherical particles, Langmuir. 10 (1994) 1439-1441, https://doi. org/10.1021/la00017a020.

[22] I.M. Kriger, T.J. Dougherty, A mechanism for non-newtonian flow in suspensions of rigid spheres, Transact. Soc. Rheol. 3 (1959) 137-152, https://doi.org/10.1122/1. 548848.

[23] H.A. Barnes, J.F. Hutton, K. Walters, An Introduction to Rheology, Elsevier, 1989.

[24] D. Santhiya, S. Subramanian, K.A. Natarajan, S.G. Malghan, Surface chemical studies on alumina suspensions using ammonium poly(methacrylate), Colloids Surf. A Physicochem. Eng. Asp. 164 (2000) 143-154, https://doi.org/10.1016/S0927-7757 (99)00347-7.

[25] J. Davies, J.G.P. Binner, The role of ammonium polyacrylate in dispersing concentrated alumina suspensions, J. Eur. Ceram. Soc. 20 (2000) 1539-1553, https://doi. org/10.1016/S0955-2219(00)00012-1.

[26] R.W. O'Brien, Electro-acoustic effects in a dilute suspension of spherical particles, J. Fluid Mech. 190 (1988) 71-86, https://doi.org/10.1017/S0022112088001211.

[27] B.J. Briscoe, A.U. Khan, P.F. Luckham, Optimising the dispersion on an alumina suspension using commercial polyvalent electrolyte dispersants, J. Eur. Ceram. Soc. 18 (1998) 2141-2147, https://doi.org/10.1016/S0955-2219(98)00147-2.

[28] P.G. de Gennes, Polymers at an interface; a simplified view, Adv. Colloid Interf. Sci. 27 (1987) 189-209, https://doi.org/10.1016/0001-8686(87)85003-0.
[29] W.B. Russel, Review of the role of colloidal forces in the rheology of suspensions, J. Rheol. 24 (1980) 287-317, https://doi.org/10.1122/1.549564.

[30] H.C. Hamaker, The London-van der Waals attraction between spherical particles, Physica. 4 (1937) 1058-1072.

[31] M. Hütter, Local structure evolution in particle network formation studied by Brownian dynamics simulation, J. Colloid Interface Sci. 231 (2000) 337-350, https://doi.org/10.1006/jcis.2000.7150.

[32] G.P. Griffin, J.J. Jurinak, Estimation of activity coefficients from the electrical conductivity of natural aquatic systems and soil extracts, Soil Sci. 116 (1973) 26-30.

[33] R. Zwanzig, R.D. Mountain, High - frequency elastic moduli of simple fluids, J. Chem. Phys. 43 (1965) 4464-4471, https://doi.org/10.1063/1.1696718.

[34] I.D. Evans, A. Lips, Concentration dependence of the linear elastic behaviour of model microgel dispersions, J. Chem. Soc. Faraday Trans. 86 (1990) 3413-3417, https://doi.org/10.1039/FT9908603413.

[35] E.C. Bingham, An investigation of the laws of plastic flow, US Bur. Stand. Bull. 13 (1916) 309-353.

[36] R. Buscall, An effective hard-sphere model of the non-Newtonian viscosity of stable colloidal dispersions: comparison with further data for sterically stabilised latices and with data for microgel particles, Colloids Surf. A Physicochem. Eng. Asp. 83 (1994) 33-42, https://doi.org/10.1016/0927-7757(93)02652-U. 